\title{
Nerve Growth Factor in the Hippocamposeptal System: Evidence for Activity-Dependent Anterograde Delivery and Modulation of Synaptic Activity
}

\author{
Lan Guo, ${ }^{1}$ Mason L. Yeh, ${ }^{2}$ Verginia C. Cuzon Carlson, ${ }^{3}$ Erin M. Johnson-Venkatesh, ${ }^{4}$ and Hermes H. Yeh ${ }^{1}$ \\ ${ }^{1}$ Department of Physiology and Neurobiology, Geisel School of Medicine at Dartmouth, Dartmouth-Hitchcock Medical Center, Lebanon, New Hampshire \\ 03756, ${ }^{2}$ Department of Cell and Developmental Biology, University College London, London WC1E 6BT, United Kingdom, ${ }^{3}$ Laboratory of Integrative \\ Neuroscience, National Institute of Alcohol Abuse and Alcoholism, Rockville, Maryland 20852, and ${ }^{4}$ Molecular and Behavioral Neuroscience Institute, \\ University of Michigan, Ann Arbor, Michigan 48109
}

Neurotrophins have been implicated in regulating neuronal differentiation, promoting neuronal survival, and modulating synaptic efficacy and plasticity. The prevailing view is that, depending on the target and mode of action, most neurotrophins can be trafficked and released either anterogradely or retrogradely in an activity-dependent manner. However, the prototypic neurotrophin, nerve growth factor (NGF), is not thought to be anterogradely delivered. Here we provide the neuroanatomical substrate for an anterograde hippocamposeptal transport of NGF by demonstrating its presence in mouse hippocampal GABAergic neurons and in their hippocamposeptal axons that ramify densely and abut neurons in the medial septum/diagonal band of Broca (MS/DB). We also demonstrate an activitydependent increase in septal NGF levels that is dependent on the pattern of intrahippocampal stimulation. In addition, we show that acute exposure to NGF, via activation of TrkA, attenuates $\mathrm{GABA}_{\mathrm{A}}$ receptor-mediated inhibitory synaptic currents and reduces sensitivity to exogenously applied GABA. These acute actions of NGF display cell type and functional selectivity insofar as (1) they were found in cholinergic, but not GABAergic, MS/DB neurons, and (2) glutamate-mediated excitatory synaptic activity as well as AMPA-activated current responses were unaffected. Our results advocate a novel anterograde, TrkA-mediated NGF signaling in the CNS.

\section{Introduction}

Neurotrophins have been implicated in myriad processes in the developing and adult nervous system. The traditional view is that neurotrophins exert influences via target-derived retrograde signaling. That is, they are produced and released by cells in target tissues, taken up by axon terminals of neurons that project there, transported retrogradely, and, within the soma, influence the expression of genes that regulate neuronal differentiation, specification, and survival (Lewin and Barde, 1996; Huang and Reichardt, 2001; Segal, 2003; Chen et al., 2005).

Nerve growth factor (NGF) is a prototypic neurotrophin in the nervous system. In the hippocamposeptal (HS) system, NGF is synthesized in hippocampal neurons and retrogradely delivered to the medial septum/diagonal band of Broca (MS/DB) (Schwab et al., 1979; Seiler and Schwab, 1984; Korsching et al., 1985; Conner and Varon, 1992; DiStefano et al., 1992; Lapchak et

Received Jan. 3, 2012; revised March 6, 2012; accepted April 5, 2012.

Author contributions: H.H.Y. designed research; L.G., M.L.Y., V.C.C.C., E.M.J.-V., and H.H.Y. performed research; L.G., M.L.Y., V.C.C.C., E.M.J.-V., and H.H.Y. analyzed data; L.G. wrote the paper.

This work was supported in part by United States Public Health Service Grants R01 NS048603 and R01 MH069826. We thank Pamela Yeh for excellent technical assistance. Caroline Nelson contributed to some of the images included in Figure 2. Walburga Croteau participated in collecting data for the experiments involving NGF ELISA.

Correspondence should be addressed to Dr. Hermes H. Yeh, Department of Physiology and Neurobiology, Geisel School of Medicine at Dartmouth, Dartmouth-Hitchcock Medical Center, Lebanon, New Hampshire 03756. E-mail: Hermes.Yeh@Dartmouth.edu.

DOI:10.1523/JNEUROSCI.0028-12.2012

Copyright $\odot 2012$ the authors $\quad 0270-6474 / 12 / 327701-10 \$ 15.00 / 0$ al., 1993; Lauterborn et al., 1993; Takeda et al., 1993; Pascual et al., 1999; Acsády et al., 2000). There, it promotes the survival of cholinergic neurons (Johnston et al., 1987; Fusco et al., 1989; Gibbs and Martynowski, 1997; Kawaja et al., 1998; Gustilo et al., 1999) and protects them from atrophic insults (Williams et al., 1986; Montero and Hefti, 1988; Junard et al., 1990; Williams, 1991). More recent studies point to an anterograde signaling scheme for neurotrophin action (Altar and DiStefano, 1998). Specifically, brain-derived neurotrophic factor (BDNF) was shown to modulate synaptic transmission as well as regulate synaptic plasticity in a fashion and time course that could best be accounted for by an anterograde signaling mechanism. In this light, current literature is replete with demonstrations of BDNF modulating, in the short term, voltage-gated ion channels and ligand-gated ionotropic receptors (Levine et al., 1996, 1998; Tanaka et al., 1997; Kafitz et al., 1999; Brünig et al., 2001; Blum et al., 2002; Cheng and Yeh, 2003; Mizoguchi et al., 2003a,b; Wardle and Poo, 2003; Fernandes et al., 2008), and, in the longer term, synaptic strength and plasticity (Kang and Schuman, 1995; Korte et al., 1998; Pozzo-Miller et al., 1999; Xu et al., 2000; Minichiello et al., 2002; Du and Poo, 2004; Pang and Lu, 2004; Bramham and Massaoudi, 2005). Importantly, the signaling pathways downstream from Trk receptor activation that confer the modulatory effects on synaptic efficacy appear to be distinct from those subserving the classic trophic actions of neurotrophins (Segal, 2003; Bramham and Massaoudi, 2005). However, for NGF, a case for it exerting neuronal effects via anterograde signaling has not been 
as compelling. Indeed, the prevailing concept is that NGF is not anterogradely transported (Altar and DiStefano, 1998).

Nonetheless, early neuroanatomical studies demonstrated that NGF mRNA is present in hippocampal GABAergic neurons, notably in the subpopulation of somatostatin-containing GABAergic neurons that projects to the MS/DB (Pascual et al., 1999; Acsády et al., 2000). It was postulated then that hippocampal GABAergic projection neurons might deliver NGF anterogradely to the MS/DB (Acsády et al., 2000). Here, we present neuroanatomical, biochemical, and electrophysiological evidence that, in toto, implicate a functional scheme for an activity-dependent anterograde mode of NGF signaling in the HS system.

\section{Materials and Methods}

All procedures involving animals were performed in accordance with the NIH Guide for the Care and Use of Laboratory Animals and approved by the Geisel School of Medicine Institutional Animal Care and Use Committee. The experiments used mice of either sex from three transgenic mouse lines: (1) the FVB-GIN (Friend virus B-type/GFP-expressing inhibitory neurons) line (Oliva et al., 2000; Jackson Laboratory); (2) the BAC-ChAT line, generated by the NINDS Gene Expression Nervous System Atlas Bacterial Artificial Chromosome (BAC) Transgenics Project (Gong et al., 2003) using a BAC vector in which the choline acetyltransferase $(C h A T)$ gene drives the expression of EGFP (obtained from Dr. Robert Brownstone, Dalhousie University, Halifax, Canada), and (3) the GAD67$\operatorname{EGFP}(\Delta$ neo) line (Tamamaki et al., 2003). In the MS/DB of the BACChAT mouse, $\sim 90 \%$ or more of the EGFP-fluorescent neurons were immunopositive for ChAT (Fig. 1A1-A3), as they were for TrkA (Fig. 1B1-B3) and p75NTR (Fig. 1C1-C3). These observations are consistent with TrkA and p75NTR being predominantly expressed in cholinergic MS/DB neurons (Sobreviela et al., 1994; Wu and Yeh, 2005). The GAD67-EGFP $(\Delta$ neo $)$ mouse line expresses EGFP regulated by the GAD67 promoter; in the mature cortex, $>80 \%$ of all GABAergic interneurons are EGFP-fluorescent (Tamamaki et al., 2003). Consistent with this, we found EGFP expressed in $86 \%$ of the parvalbuminimmunoreactive subpopulation of GABAergic MS/DB neurons (data not shown). The EGFP-fluorescent cells in the BAC-ChAT and GAD67$\operatorname{EGFP}(\Delta$ neo $)$ mice greatly facilitated identification of cholinergic and GABAergic MS/DB neurons in the acute brain slices used in our electrophysiological experiments. Figure $5 A 2$ illustrates two EGFP-fluorescent neurons in the MS/DB of an acute brain slice obtained from the BACChAT mouse.

Immunohistochemistry. Postnatal day 21-28 (P21-P28) mice were asphyxiated with carbon dioxide and immediately perfused intraaortically with heparinized PBS followed by $4 \%$ paraformaldehyde/ $0.1 \mathrm{M}$ PBS. Brains were isolated, postfixed overnight in $4 \%$ paraformaldehyde, and then immersed sequentially in $0.1 \mathrm{M}$ PBS containing 15 and $30 \%$ sucrose. Cryosections $(40 \mu \mathrm{m})$ containing the MS/DB or hippocampus were blocked in $10 \%$ normal goat serum $/ 0.05 \%$ Triton X-100/PBS before incubation with primary or secondary antibodies. The primary antibodies used were mouse anti-GFP (1:1000; Invitrogen), rabbit anti-NGF (1:1000; Sigma-Aldrich), mouse antiparvalbumin (1:1000; Millipore), rabbit anti-ChAT (1:1000; Millipore), rabbit anti-TrkA (1:1000; Millipore), and rabbit anti-p75NTR
(1:1000; Millipore). Secondary antibodies used were Alexa Fluor 350, Alexa Fluor 488, Alexa Fluor 555, and Alexa Fluor 568 (Invitrogen) diluted 1:800 in PBS. Sections were coverslipped in FluorSave Reagent (Calbiochem). In one series, the HS system was microdissected intact and $40 \mu \mathrm{m}$ cryosections with contiguous hippocampus, HS pathway, and MS/DB were processed for immunohistochemistry (see Fig. $2 \mathrm{~A}$ ). Immunofluorescent images were captured using a spinning disc confocal microscope (BX61, Olympus), some images were deconvoluted and edited using Photoshop CS (Adobe). Negative control sections with the primary antibody omitted were routinely run in parallel.

Intact HS preparation. Assessing septal NGF levels following hippocampal stimulation called for a preparation in which the connection between the two structures is preserved. To this end, the hippocampus, the HS pathway, and the septum were dissected intact from brains isolated from P21-P28 mice and placed in a custommade two-compartment chamber, immersed in oxygenated artificial CSF (aCSF) identical to that used for electrophysiological recording (see below), and maintained at $35-37^{\circ} \mathrm{C}$ (see Fig. $4 \mathrm{~A}$ ). The hippocampus was placed in one compartment and the septum in the other. Vaseline placed between the compartments and embedding the HS pathway served as a barrier so that solution exchanges and electrical stimulation could be achieved in one compartment without affecting the adjoining one. Routinely, the hippocampus of the HS preparation obtained from one side of the brain was either exposed to $\mathrm{KCl}$ ( 25 $\mathrm{mm}$ ) or stimulated using a pair of bipolar electrodes for $15 \mathrm{~min}$, after which the septum was detached and processed for NGF-ELISA (see below). As control, the HS preparation from the contralateral side of the brain was incubated in aCSF for the same length of time without electrical stimulation or $\mathrm{KCl}$-induced depolarization. 


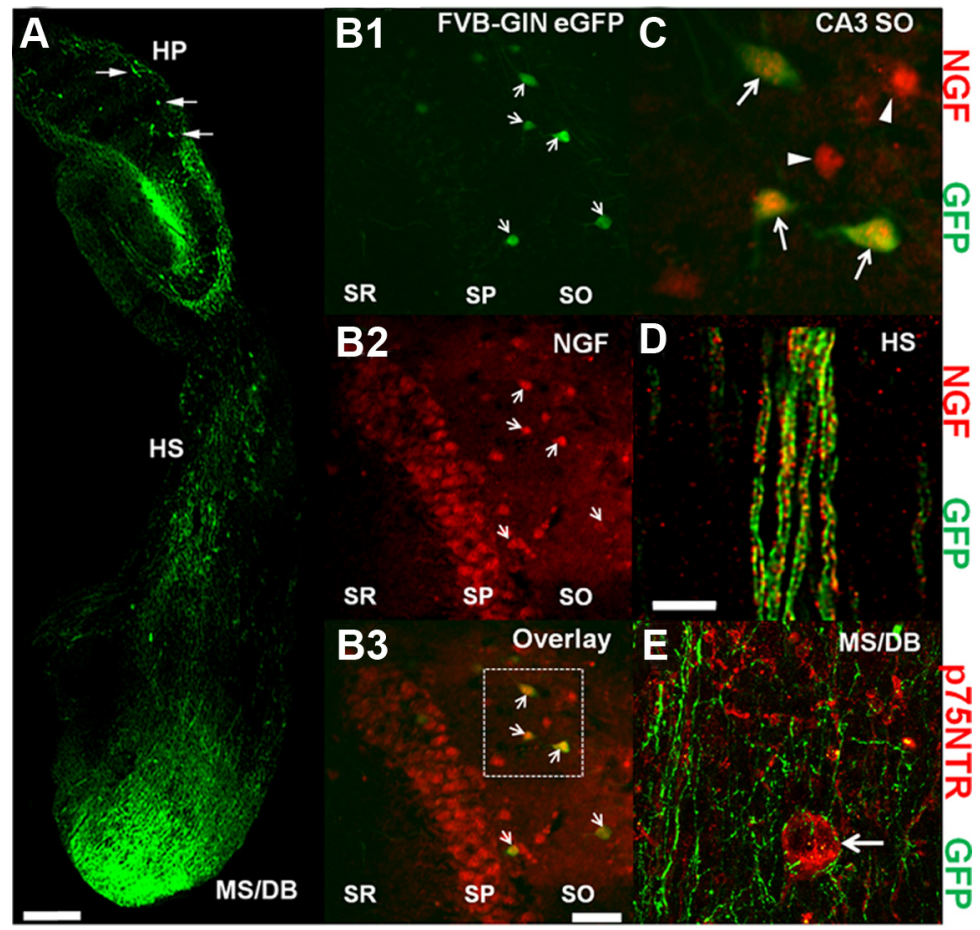

Figure 2. Hippocamposeptal projecting GABAergic neurons contain NGF. A, Cryosection containing contiguous hippocampus and MS/DB derived from the FVB-GIN mouse brain illustrating EGFP-fluorescent hippocampal neurons (arrows) and axons emanating from the hippocampus (HP) and running along the hippocamposeptal pathway to ramify densely in the MS/DB. Scale bar, $1 \mathrm{~mm}$. $\boldsymbol{B}$, Representative field taken from the CA3 region of FVB-GIN hippocampus showing EGFP-immunopositive (B1, arrows) and NGF-immunopositive ( $\boldsymbol{B} 2$, arrows) neurons. The overlay ( $\boldsymbol{B} 3$ ) reveals that many of the EGFP-containing neurons are immunoreactive for NGF (arrows). Scale bar, $50 \mu \mathrm{m}$. C, Higher magnification image showing overlay of EGFP (green) and NGF (red) immunoreactivity in CA3 stratum oriens (B3, boxed area). EGFP-expressing neurons (arrows) are a subset of those that are NGF-immunoreactive as some NGF-immunoreactive neurons do not express EGFP (arrowheads). $\boldsymbol{D}$, EGFP-immunopositive HS fibers in the FVB-GIN mice are decorated by punctate NGF-immunoreactive profiles. $\boldsymbol{E}$, In MS/DB, EGFP-immunoreactive fibers (green) course around and come into close contact with a p75NTR-immunoreative cholinergic neuron (red, white arrow). Negative controls (parallel processing without primary antibody) revealed no nonspecific immunostaining. HP, hippocampus; SR, CA3 stratum radiatum; $S P$, CA3 stratum pyramidale; SO, CA3 stratum oriens. Scale bar: (in D) $\mathbf{C}-\boldsymbol{E}, 20 \mu \mathrm{m}$.

NGF-ELISA. NGF sandwich ELISA was performed using NGF Emax ImmunoAssay System (Promega). Briefly, ELISA plates (Costar) coated with anti-NGF polyclonal antibody were incubated overnight at $4^{\circ} \mathrm{C}$ and then blocked at room temperature for $1 \mathrm{~h}$ using blocking and sampling buffer. Supernatant from homogenate of dissected septum containing the MS/DB and hippocampus were diluted in blocking and sampling buffer, added into the wells of ELISA plates along with NGF standards, and incubated at room temperature for $6 \mathrm{~h}$. Anti-NGF monoclonal antibody was then added and the samples were allowed to incubate overnight at $4^{\circ} \mathrm{C}$. Anti-rat IgG HRP conjugate was then added at room temperature for $2.5 \mathrm{~h}$. Color development was achieved by adding TMB One Solution and terminated by adding $1 \mathrm{~N} \mathrm{HCl}$. The plates were read at $450 \mathrm{~nm}$ (Bio-Rad), and a standard curve was constructed using murine NGF standards to derive estimates of NGF content in hippocampal and MS/DB samples. Data were reported as mean \pm SEM. Comparative analysis of NGF levels in the septum and hippocampus without and with hippocampal stimulation was performed using Student's $t$ test.

Electrophysiology. Mice (P10-P22) were asphyxiated by carbon dioxide and decapitated. The brain was quickly isolated, blocked by removing the cerebellum and hindbrain, and immersed en bloc in ice-cold oxygenated aCSF containing (in $\mathrm{mm}$ ) the following: $125 \mathrm{NaCl}, 2.5 \mathrm{KCl}, 2.0$ $\mathrm{CaCl}_{2}, 1.0 \mathrm{MgCl}_{2}, 1.25 \mathrm{NaH}_{2} \mathrm{PO}_{3}, 26 \mathrm{NaHCO}_{3}$, and 10 glucose. All solutions were bubbled continuously with $5 \% \mathrm{CO}_{2}$ and $95 \% \mathrm{O}_{2}$. Coronal brain slices $(200 \mu \mathrm{m})$ containing MS/DB were obtained using a vibrating tissue slicer (Model OTS-4000, Electron Microscopy Sciences). The slices were incubated in aCSF at room temperature for at least $60 \mathrm{~min}$ before electrophysiological recording.
During electrophysiological recording, the brain slices were maintained at $32-33^{\circ} \mathrm{C}$ on a heated stage fit onto an upright microscope (BXWI50, Olympus) and continuously perfused with oxygenated aCSF at rate of $0.5 \mathrm{ml} /$ min. A CCD camera attached to a video frame grabber board (Integral Technologies) displayed real-time images to facilitate visualization of cells under Hoffman Modulation Optics and placement of recording electrodes and drug pipettes. In slices derived from wildtype mice, putative cholinergic and noncholinergic MS/DB neurons were identified in the cell-attached mode by firing rate $\mathrm{Wu}$ and $\mathrm{Yeh}$, 2005). In slices derived from the BAC-ChAT mice, cholinergic neurons were readily identifiable by their expression of fluorescent EGFP (Fig. 1).

Patch electrodes (2-6 M $\Omega$ in recording solution) were pulled from borosilicate glass capillary tubes (outer diameter, $1.5 \mathrm{~mm}$; inner diameter, $0.86 \mathrm{~mm}$ ). The internal (recording) solution used consisted of the following (in mM): $140 \mathrm{KCl}, 4 \mathrm{NaCl}, 2 \mathrm{MgCl}_{2}, 10$ EGTA, 10 HEPES, $2 \mathrm{Mg}$-ATP, and $3 \mathrm{Mg}$-GTP, pH 7.3, with $\mathrm{KOH}$. Some experiments used an internal solution consisting of the following (in $\mathrm{mM}$ ): 144 K-gluconate, $3 \mathrm{MgCl}_{2}, 0.2$ EGTA, 10 HEPES, $3 \mathrm{Mg}$-ATP, 3Na-GTP, and 1 leupeptin, $\mathrm{pH}$ 7.3, with KOH. For recordings of inhibitory synaptic currents, the NMDA receptor antagonist 2-amino-5-phosphonovaleric acid (APV; $10 \mu \mathrm{M}$; Tocris Bioscience) and AMPA receptor antagonist 6-cyano-7-nitroquinoxaline-2,3dione (CNQX; $10 \mu \mathrm{m}$; Tocris Bioscience) were added to the aCSF perfusion solution to block excitatory synaptic currents. Under these conditions, bicuculline methiodide ( $10 \mu \mathrm{M}$; Tocris Bioscience) reversibly abolished all synaptic currents (data not shown). For recordings of excitatory synaptic currents, the $\mathrm{GABA}_{\mathrm{A}}$ receptor antagonist bicuculline methiodide $(10 \mu \mathrm{M})$ or gabazine (10 $\mu \mathrm{M}$, Sigma-Aldrich) was added to the perfusion solution. Membrane currents were monitored using a multiclamp 700B amplifier (Molecular Devices), filtered at $5 \mathrm{kHz}$, digitized using Pulse and analyzed off-line using PulseFit (HEKA Instruments), Mini Analysis software (Version 6.0.7, Synaptosoft), or Clampfit software (Molecular Devices). Statistical analysis was performed using SigmaPlot 8.02 (SPSS) and Prism (Version 5, GraphPad Software). Mean peak current and frequency of GABA-mediated spontaneous synaptic events were analyzed using paired Student's $t$ test. Repeated-measures ANOVA and Bonferroni's multiple-comparison test were applied when comparing NGF treatment to baseline and washout in the same cell. Data were reported as mean \pm SEM.

Applications of drugs and extracellular stimulation. Unless indicated otherwise, all chemicals were purchased from Sigma-Aldrich. NGF $(2.5 \mathrm{~S}$ NGF, Harlan), bicuculline methiodide, gabazine, APV, CNQX, GABA, and AMPA were dissolved in aCSF, stored as frozen stock and diluted to working concentrations with aCSF immediately before each recording session.

For focal application, the drug solutions were loaded into separate barrels of a six-barrel pipette assembly mounted onto a micromanipulator and navigated under microscopic control to be within $10 \mu \mathrm{m}$ of the soma of the MS/DB neuron under study (Wu and Yeh, 2005; see Fig. $5 A 1)$. Drug solutions were ejected by regulated pulses of pressure $(\leq 3$ psi; Picospritzer, General Valve Company), with timing and duration controlled by a digital timing unit (Pulsemaster A300, World Precision Instruments). One barrel was routinely filled with external solution, which was applied between drug applications to clear drugs from the 
vicinity of the cell and to control for mechanical artifacts due to bulk flow.

Extracellular stimulation was performed with a concentric bipolar electrode (Frederick Haer) inserted into one aCSF-filled barrel of the drug pipette assembly, placed $\geq 50 \mu \mathrm{m}$ from the soma under study. The A360 Stimulus Isolator (World Precision Instruments) was used to generate the current pulses triggered by the digitizer. Stimulus intensity was adjusted in each case to evoke stable postsynaptic currents in the cell recorded and then kept constant throughout the recording.

\section{Results}

We addressed three questions: (1) Is NGF localized to GABAergic hippocampal neurons and their axons that project to the MS/DB? (2) Does stimulation of the hippocampus increase NGF levels in the MS/DB? (3) Does NGF modulate inhibitory and/or excitatory synaptic activity in the MS/DB?

\section{NGF is present in hippocampal GABAergic neurons that project to the MS/DB}

In the hippocampus and neocortex of the FVB-GIN (Friend virus B-type/GFP-expressing inhibitory neurons) mouse, a subpopulation of somatostatin-containing GABAergic neurons express EGFP (Oliva et al., 2000). As illustrated in Figure 2 A, EGFPimmunopositive fibers course along the HS pathway and ramify densely in the MS/DB. These fibers are axons of GABAergic neurons in the hippocampus (Fig. $2 A$, arrows) since GABAergic neurons native to the MS/DB do not express EGFP (Oliva et al., 2000). NGF-like immunoreactive profiles could be found in all strata and cornu ammonis (CA) regions of the hippocampus. Figure $2 \mathrm{~B} 1$ shows EGFP immunoreactive GABAergic neurons in the CA3 region of the hippocampus (arrows), many of which coexpress NGF (Fig. 2 B2,B3, arrows). The boxed area in Figure $2 B 3$ is shown at higher magnification in Figure $2 C$ to illustrate that NGF immunoreactivity in the stratum oriens was not limited to the EGFP-immunofluorescent GABAergic subpopulation (Fig. 2C, arrows) but was also found in non-EGFP-fluorescent cells (Fig. 2C, arrowheads). These likely represent other subtypes of GABAergic neurons that express NGF mRNA in the hippocampus (Pascual et al., 1999). In addition, punctate NGFimmunoreactive profiles could be found along the length of the EGFP-containing axons in the HS pathway (Fig. 2D) and in the MS/DB (data not shown). In the MS/DB, these axons ramified densely, frequently outlining closely the contours of neurons, including virtually all cholinergic neurons identified by their expression of p75NTR immunoreactivity (Fig. 2 E, arrow; Heckers et al., 1994; Sobreviela et al., 1994). This is in agreement with an earlier report that GABAergic hippocampal neurons innervate cholinergic MS/DB neurons, albeit to a lesser extent than their GABAergic counterpart (Tóth et al., 1993). Overall, these findings established the neuroanatomical substrate for an anterograde mode of NGF delivery in the HS system. We also noted many NGF-immunopositive cellular profiles in the MS/DB (Fig. 3). Presumably, many are GABAergic MS/DB neurons (Conner and Varon, 1992; Lauterborn et al., 1995), as indicated by colocalization of NGF with parvalbumin-immunoreactivity (Fig. $3 A 1, A 3, B$, arrowheads), or ChAT-immunoreactive cholinergic neurons, with retrogradely transported NGF sequestered in the soma (Fig. 3A1,A2,B, arrow), and others may be nonneuronal elements, such as astrocytes.

Stimulation of the hippocampus increases NGF levels in the MS/DB in a pattern-dependent manner

Using an intact HS preparation (Fig. $4 A$, inset), we asked whether stimulation of the hippocampus could increase the level of NGF

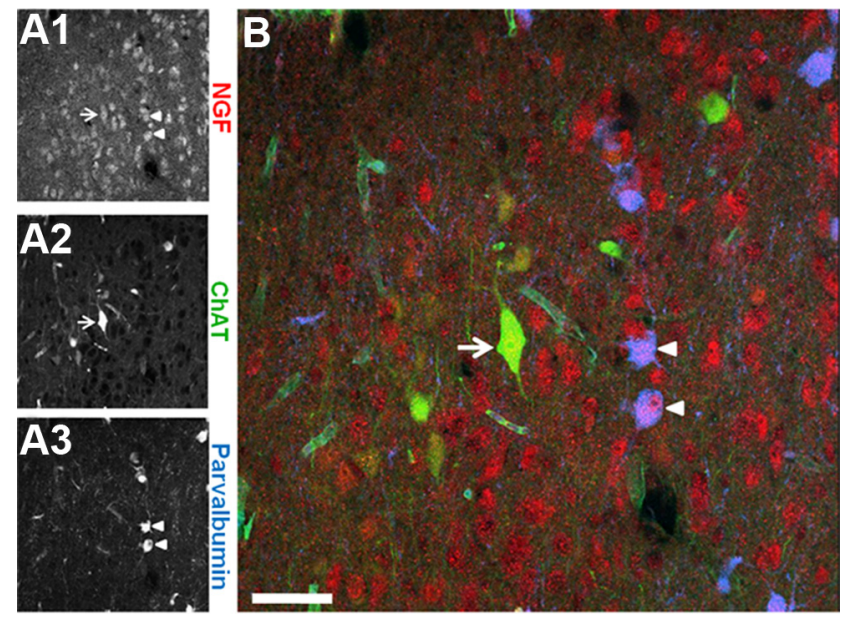

Figure 3. MS/DB neurons display NGF-like immunoreactivity. A1, NGF immunostaining in the MS/DB. Arrow and arrowheads point to three representative cells that display NGF-like immunoreactivity. A2, ChAT immunostaining in the same field as A1. Arrow points to a ChATimmunoreactive cholinergic MS/DB neuron. $A 3$, Parvalbumin immunostaining in the same field as $\boldsymbol{A 1}$. Arrowheads point to two parvalbumin-immunoreactive GABAergic MS/DB neurons. $\boldsymbol{B}$, Overlay of $\boldsymbol{A} \mathbf{1}-\boldsymbol{A} \mathbf{3}$ showing colocalization of NGF-like immunoreactivity in cholinergic (arrow) and GABAergic (arrowheads) MS/DB neurons. Scale bar: $\boldsymbol{B}, 40 \mu \mathrm{m}$.

in the MS/DB. Since the boundaries of the medial and lateral septa and diagonal band of Broca were not sufficiently demarcated to allow further microdissection in this preparation, the septum was isolated en bloc (Fig. 4A, black arrow; Fig. 4A inset, black arrowhead). The hippocampal portion of the HS preparation was subjected to either constant depolarization with $\mathrm{KCl}$ ( 25 $\mathrm{mm}$ ) or, in an attempt to simulate firing patterns of different hippocampal neuronal subpopulations, one of three patterns of electrical stimulation: (1) a burst-type pattern (24 Hz pulses delivered as trains of eight pulses at $100 \mathrm{~Hz}$ at 300 ms intervals; 500 $\mu \mathrm{A})$, simulating the fast firing pattern of somatostatinimmunopositive GABAergic neurons in the hippocampus and cortex (Freund and Buzsáki, 1996; McBain and Fisahn, 2001), which frequently occurred in bursts, as reported in the frontal cortex (Kawaguchi and Kubota, 1996); (2) an evenly spaced barrage of the same number of single pulses delivered at $42 \mathrm{~ms}$ intervals $(24 \mathrm{~Hz} ; 500 \mu \mathrm{A})$, simulating the general firing characteristics of hippocampal GABAergic interneurons (Freund and Buzsáki, 1996; McBain and Fisahn, 2001), and (3) evenly spaced pulses $(500 \mu \mathrm{A})$ delivered at $3 \mathrm{~Hz}$, simulating the activity of other neuronal types in the hippocampus with low rates of spontaneous firing. Following $15 \mathrm{~min}$ of stimulation, the hippocampus and septum were detached and processed separately to determine NGF levels by ELISA. The HS preparation obtained from the left hemisphere was routinely used for stimulation; that obtained from the right hemisphere was used as no-stimulation control.

The scatter plot in Figure $4 B$ illustrates ELISA data from 30 separate experiments in which septal NGF levels were assessed following various forms of hippocampal stimulation. Each point represents an individual experiment and denotes the NGF level in the septum following hippocampal stimulation relative to that obtained in the control counterpart. The equivalence line (dashed line) represents the result expected if NGF levels in the septum remained unchanged following hippocampal stimulation. All of the points representing the individual experiments in which the hippocampus was stimulated using a burst pattern (gray diamonds) were positioned above the equivalence line, in- 

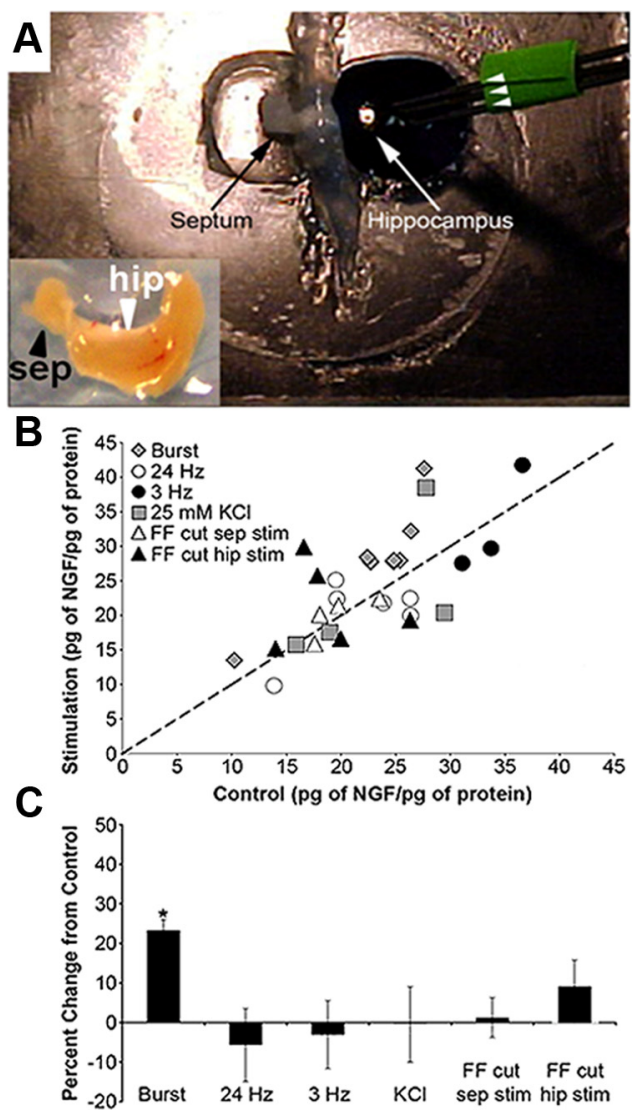

Figure 4. Intact hippocamposeptal preparation and experimental setup for demonstrating stimulus-evoked NGF levels in hippocamposeptal system. $\boldsymbol{A}$, Custom-made temperaturecontrolled two-compartment incubating chamber holding the intact septohippocampal preparation (inset). The hippocampus was placed in one compartment and the septum in the other. A strip of Vaseline positioned between the compartments and embedding the hippocamposeptal pathway permitted solution exchange and stimulation in individual compartments. To demonstrate that the two chambers are isolated, methyl green (1\%) added to the compartment holding the hippocampus (right) does not leak into the left compartment holding the septum. Three bipolar stimulating electrodes (triple arrowheads) aligned in parallel are shown inserted into the hippocampus. B, Scatterplot summarizing septal NGF levels determined by ELISA. In experiments with the intact septohippocampal preparation, the hippocampus was stimulated for 15 min with a train of eight pulses at $100 \mathrm{~Hz}$ delivered at 300 ms intervals (Burst, gray diamond), single regularly spaced pulses at $24 \mathrm{~Hz}$ (open circle) and $3 \mathrm{~Hz}$, filled circle), and $\mathrm{KCl}$ (25 $\mathrm{mm}$, gray square). In experiments with the fimbria/fornix severed, either the septum (FF cut sep stim, open triangle) or the hippocampus (FF cut hip stim, filled triangle) was stimulated with the burst-type pattern stimulation for $15 \mathrm{~min}$. Each symbol represents data obtained from one experiment. The $45^{\circ}$ equivalence line (dotted line) represents the result expected if the NGF level remains unchanged after stimulation compared with no-stimulation control. C, Mean percentage change from control of septal NGF levels using stimulation paradigms as indicated. Data expressed as mean \pm SEM. HP, hippocampus; SEP, septum.

dicating consistently increased levels of NGF in the septum. As summarized in Figure 4C, patterned burst stimulation of the hippocampus increased NGF levels in the septum by $23.3 \pm 4.6 \%$ $(N=7 ; p<0.05)$.

By contrast, electrical stimulation with single, evenly spaced 24 or $3 \mathrm{~Hz}$ pulses (Fig. 4 B, open and filled circles, respectively), or with constant $\mathrm{KCl}$-induced depolarization (Fig. 4B, gray squares), were ineffective in increasing NGF levels in the septum; the data points tended to cluster around the equivalence line. As summarized in Figure $4 C$, stimulation with single, evenly spaced pulses at $24 \mathrm{~Hz}(-4.4 \pm 24.8 \% ; N=4 ; p=0.82)$ and at $3 \mathrm{~Hz}$ $(-3.1 \pm 8.6 \% ; N=3 ; p=0.54)$ yielded statistically insignificant outcomes. In the hippocampi of the same preparations, there was a trend toward elevated NGF levels following the three patterns of electrical stimulation, but this turned out to be statistically insignificant (data not shown).

We asked whether the stimulus-evoked increase in septal NGF levels was dependent on an intact HS pathway. To address this, we severed the fimbria/fornix in the HS preparation and found that intrahippocampal stimulation with the bursttype pattern no longer elevated septal NGF levels (Fig. 4C). In addition, despite demonstration of NGF endogenous to the MS/DB (Fig. 3), septal stimulation failed to elevate NGF levels in the septum (Fig. 4C). These results point to increased NGF levels in the septum following patterned stimulation of the hippocampus.

\section{NGF modulates inhibitory but not excitatory neurotransmission in cholinergic MS/DB neurons}

Our findings that NGF is present in axons of HS GABAergic neurons and that intrahippocampal stimulation increases septal NGF levels are consistent with there being an anterograde route of NGF delivery to the MS/DB neurons. Since extracellularly applied NGF excites cholinergic MS/DB neurons (Wu and Yeh, 2005), we hypothesized that the neurotrophin could do so by modulating GABA and/or glutamate receptor function in cholinergic MS/DB neurons, GABAergic MS/DB neurons, or both.

We first assessed whether NGF affected GABA-activated current responses in cholinergic neurons in acute brain slices derived from wild-type, BAC-ChAT, and GAD67-EGFP( $\Delta$ neo $)$ mice. In Figure $5 A 1$, the recording pipette $(\mathrm{R})$ is pointed at an EGFPexpressing MS/DB neuron (Fig. 5A2) in a brain slice from a BACChAT mouse. The multibarrel drug pipette assembly for focal application of drugs $(\mathrm{P})$ is positioned near the neuron under recording. Pressure-regulated pulses of GABA $(20 \mu \mathrm{M} ; 200-600$ $\mathrm{ms}$ duration) were applied at $20 \mathrm{~s}$ intervals. With aCSF applied throughout the length of the interpulse interval, the GABA responses remained stable and did not run down for the duration of the recording session. Whole-cell current responses to GABA were monitored continuously before, during, and after NGF (100 $\mathrm{ng} / \mathrm{ml}$ ) application (Fig. 5B1-B3). For each cell, the peak amplitude of six consecutive responses recorded under each condition were averaged and used for quantitative comparison. Figure $5 C$ illustrates superimposed examples of such averaged responses before, during, and after NGF exposure obtained from the same cell. Repeated-measures ANOVA revealed a significant effect of acute NGF exposure on the peak amplitude of the GABA response (Fig. $5 D ; n=12 ;{ }^{*} p=0.003$ ); post hoc Bonferroni's multiple-comparison test indicated that the peak amplitude of the GABA response during NGF application $(81.02 \pm 17.52 \mathrm{pA})$ was significantly reduced compared with control period (110.4 \pm $\left.23.36 \mathrm{pA} ;{ }^{*} p<0.05\right)$. In two cells, focal application of K252a (10 min) prevented NGF-induced suppression of $\mathrm{GABA}_{\mathrm{A}}$ receptormediated current responses (data not shown), consistent with the involvement of TrkA signaling in mediating the NGF-induced suppression of GABA responses.

We then asked whether NGF alters GABAergic synaptic transmission in the MS/DB. We recorded spontaneous $\mathrm{GABA}_{\mathrm{A}^{-}}$ mediated IPSCs (sIPSCs) from cholinergic MS/DB neurons in acute coronal slices derived from the BAC-ChAT mice. Representative sIPSCs recorded from a cholinergic MS/DB neuron before and during exposure to focally applied NGF are illustrated in Figure 6A. Before NGF exposure, the mean amplitude of the sIPSCs was $50.3 \pm 6.7 \mathrm{pA}$ (Fig. $6 \mathrm{~B}$, open bar). Upon a $2 \mathrm{~min}$ exposure to NGF, there was a significant, albeit modest, decrease 
in the mean amplitude of the sIPSCs (Fig. $6 B$, striped bar; $46.7 \pm 5.8 \mathrm{pA} ; n=12$; paired $t$ test, $p=0.03)$. Application of NGF did not alter the mean frequency of IPSCs (Fig. 6C; $n=12$; paired $t$ test, $p=$ $0.29)$.

In another set of experiments, electrical stimuli $(0.5 \mathrm{~ms}$ duration, $500 \mathrm{~ms}$ intervals) were delivered to the vicinity of cholinergic MS/DB neurons. The mean amplitude of 20 evoked IPSCs (eIPSCs) obtained during the control (pre-NGF) period was compared with that obtained during a $2 \mathrm{~min}$ focal application of NGF and that following a 5 min clearing of the neurotrophin with continuous perfusion of aCSF. Representative averaged traces obtained from a cell under each of the three conditions are superimposed and illustrated in Figure 6G. NGF application significantly decreased the mean amplitude of eIPSCs (Fig. $6 H ; n=9$, repeated-measures ANOVA, $p<0.01)$. Specifically, the mean amplitude of eIPSCs during NGF application (40.64 \pm 9.85 pA) was significantly lower compared with the control $(47.26 \pm 10.04 \mathrm{pA})$ and wash periods $(44.29 \pm 10.36 \mathrm{pA})$ (Fig. $6 H$; Bonferroni's multiple-comparison tests, $p<0.05)$. The above results suggest that NGF-TrkA signaling suppresses $\mathrm{GABA}_{\mathrm{A}}$-receptor-mediated neurotransmission in cholinergic $\mathrm{MS} / \mathrm{DB}$ neurons.

Unlike its suppression of GABA-activated responses, NGF did not affect the amplitude of AMPA $(20 \mu \mathrm{M})$-activated current responses in cholinergic $\mathrm{MS} / \mathrm{DB}$ neurons $(n=9$, repeatedmeasures ANOVA, $p>0.05)$. The mean amplitude of the wholecell current responses to AMPA before NGF exposure (control; $53.06 \pm 8.39 \mathrm{pA}$ ) was not statistically different from that during NGF application $(60.92 \pm 11.68 \mathrm{pA})$ nor that following washout $(52.51 \pm 7.83 \mathrm{pA})$. Focal application of NGF also did not alter spontaneous EPSCs (sEPSCs) in cholinergic MS/DB neurons. In 20 cholinergic neurons, the mean sEPSC amplitude and frequency during the control period were $18.07 \pm 1.31 \mathrm{pA}$ and $1.50 \pm 0.72 \mathrm{~Hz}$, respectively. These values remained unchanged upon NGF application (mean amplitude, $17.25 \pm 1.24 \mathrm{pA}$; mean frequency, $1.45 \pm 0.66 \mathrm{~Hz}$; paired $t$ test, $p>0.05$ ). Similarly, NGF had no effect on evoked EPSCs (eEPSCs) in cholinergic MS/DB neurons. The mean eEPSC amplitude during NGF application was $40.16 \pm 5.90 \mathrm{pA}$, which was not significantly different from that recorded before or after NGF exposure $(41.45 \pm 5.64 \mathrm{pA}$ and $39.90 \pm 6.51 \mathrm{pA}$, respectively; $n=6$, repeated-measures ANOVA, $p>0.05)$. Together, these results indicate that NGF preferentially modulates inhibitory vis-à-vis excitatory neurotransmission in the MS/DB.

Is the modulatory effect of NGF specific to the cholinergic neurons in the MS/DB? To answer this question, we examined sIPSCs (Fig. 6D-F) and sEPSCs (data not shown) in EGFPfluorescent MS/DB neurons from the $G A D 67-\operatorname{EGFP}(\Delta$ neo $)$ as well as non-EGFP-fluorescent MS/DB neurons from the BACChAT mice. These cells typically displayed a fast rate of firing consistent with them being noncholinergic NS/DB neurons, as reported previously (Wu and Yeh, 2005). Acutely exposing these neurons to NGF did not affect the amplitude or frequency of
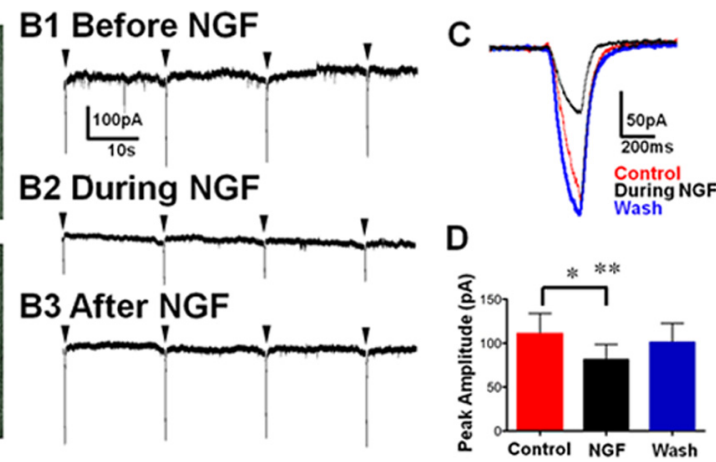

Figure 5. NGF decreases GABA-evoked whole-cell current responses in cholinergic MS/DB neurons. $A$, EGFP-fluorescent MS/DB neurons viewed under Hoffman Modulation 0ptics $(\boldsymbol{A 1})$ and epifluorescence $(\boldsymbol{A 2})$ in an acute coronal brain slice containing the 列 作 attenuates responses to exogenously applied GABA as reflected in a decrease in the peak amplitude of the current responses during NGF application (repeated-measures ANOVA, ${ }^{* *} p<0.01$; Bonferroni's multiple-comparison test, ${ }^{*} p<0.05$ ).

sIPSCs (Fig. 6E, $F ; n=9$ ) nor of $\operatorname{sEPSCs}(n=6)$ (paired $t$ test, $p>$ 0.05). These results indicate that NGF not only preferentially modulates postsynaptic $\mathrm{GABA}_{\mathrm{A}}$ receptor function, but also does so selectively in the TrkA-expressing cholinergic MS/DB neurons (see Fig. 1B1-B3).

\section{Discussion}

The mode of neurotrophin transport bears important functional implications. Thus, the traditional scheme of retrogradely transported target-derived NGF being sequestered in the soma and enhancing the expression of genes and factors that promote neuronal differentiation, phenotypic specification, and survival provided the framework for marshaling the classical "neurotrophin hypothesis" (Davies, 1996). Along the same line, the demonstration that certain neurotrophins, notably BDNF and NT3, are in synaptic terminals of brain regions where the corresponding $\mathrm{mR}$ NAs are absent prompted the notion of anterograde transport, which in turn provided a scheme for their activity-dependent release and extracellular accumulation in modulating synaptic efficacy and plasticity (Altar et al., 1997; Conner et al., 1997; Yan et al., 1997; Schinder and Poo, 2000). To date, evidence for activity-dependent release of NGF and its effect on synaptic plasticity is piecemeal at best (Blöchl and Thoenen, 1995, 1996; Wu et al., 2004).

Our study combined immunohistochemical, ELISA, and electrophysiological analyses to determine whether NGF synthesized in the hippocampus could signal in an anterograde fashion to modulate synaptic transmission in target MS/DB neurons. The data presented here indicate for the first time that NGF in the HS system fulfills many of the prerequisite criteria for a functional anterograde mode of delivery and activity-dependent release, placing NGF in a favorable position to modulate synaptic transmission. The major findings are as follows: (1) HS GABAergic neurons display NGF-like immunoreactivity, which can be traced along their axons en route to and within the MS/DB; (2) hippocampal stimulation rapidly elevates septal NGF levels in a stimulus pattern-dependent fashion; and (3) NGF selectively attenuates inhibitory neurotransmission in TrkA receptor-expressing cholinergic MS/DB neurons. 


\section{A Cholinergic}

Before NGF

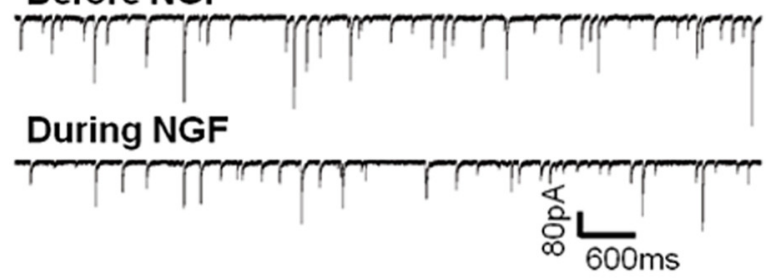

B Cholinergic

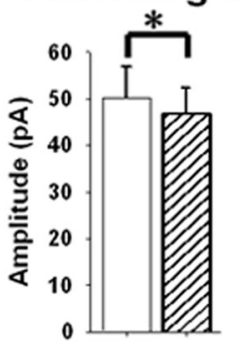

\section{Cholinergic}

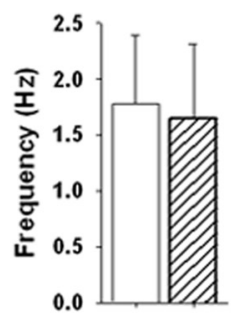

D Non-cholinergic

Before NGF

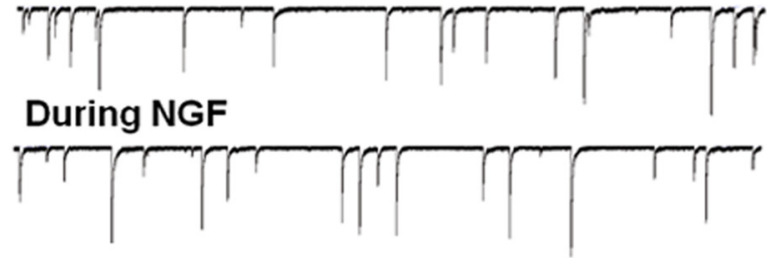

E Non-cholinergic F Non-cholinergic
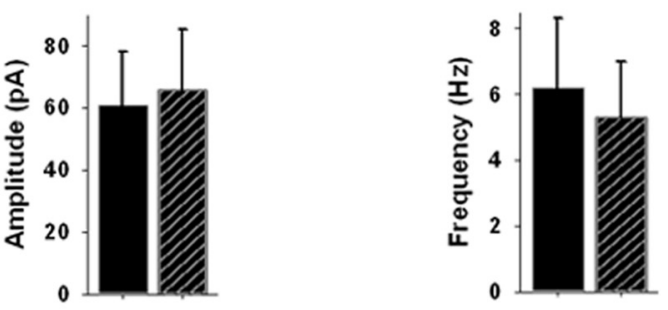

G Evoked IPSCs

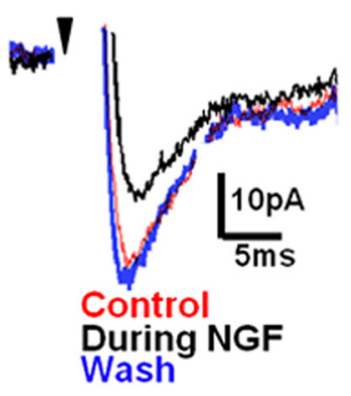

H Evoked IPSCs

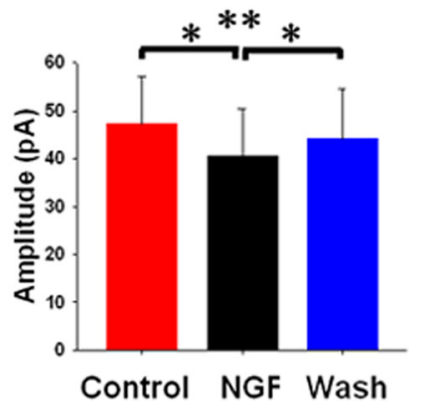

Figure 6. NGF attenuates inhibitory synaptic activity in cholinergic MS/DB neurons. A, SIPSCS recorded in the presence of CNQX and APV (10 $\mu \mathrm{m}$ each) in a cholinergic MS/DB neuron before and during focal application of NGF. $\boldsymbol{B}$, Histogram summarizing the amplitude of SIPSCS before NGF (open bars) and during NGF (striped bars) in cholinergic MS/DB neurons. The mean amplitude of sIPSC $s$ was significantly decreased upon NGF application (paired $t$ test, $p<0.005$ ). C, Summary of the frequency of sIPSCs before NGF (open bars) and during NGF (striped bars) obtained from cholinergic MS/DB neurons. NGF did not significantly alter sIPSC frequency (paired $t$ test, $p>0.005$ ). $\boldsymbol{D}-\boldsymbol{F}$, The same sets of experiments performed to assess NGF effects

NGF expression in the HS system

The FVB-GIN mouse line (Oliva et al., 2000) facilitated the examination of NGF expression in the EGFP-fluorescent subpopulation of somatostatin-containing GABAergic neurons that project from the hippocampus to the MS/DB. Beside being localized to EGFPexpressing cells, NGF-immunoreactivity in the hippocampus proper was also localized to other nonpyramidal cells, presumably GABAergic interneurons expressing other peptidergic or calcium-binding protein markers (Freund and Buzsáki, 1996). Our findings corroborate earlier reports localizing NGF mRNA to hippocampal neurons immunoreactive for a number of neuropeptides, including somatostatin, neuropeptide-Y, vasoactive intestinal polypeptide, and cholecystokinin (Pascual et al., 1999).

Oliva et al. (2000) speculated that the EGFP-expressing neurons projected extrahippocampally. Here, as revealed in cryosections with the HS system intact, we demonstrate unequivocally such a projection from the hippocampus proper via the fornix to the MS/DB. Since we never observed EGFP-expressing cell bodies in the MS/DB of FVB-GIN mice, the dense EGFP-fluorescent fibers seen along the HS pathway and in the MS/DB must have been axons of GABAergic neurons residing in the hippocampus. Furthermore, punctate NGF-immunoreactive profiles were present within these axons through the entire extent of the projection. In line with an earlier report that GABAergic hippocampal neurons innervate the cholinergic MS/DB neurons (Tóth et al., 1993), we found in the FVB-GIN mice EGFP-expressing axons outlining the contours of many neurons in the MS/DB, including the cholinergic neurons immunolabeled by p75NTR. Overall, these observations provided the prerequisite neuroanatomical basis to assess stimulus-dependent release and modulation of synaptic activity in the MS/DB.

\section{NGF levels in the MS/DB: dependence on} intrahippocampal stimulation

Intrahippocampal stimulation elevated NGF levels in the MS/DB in an acute intact HS preparation. The hippocampus was the source of the increased NGF level since, with the fimbria/fornix severed, the intrahippocampal stimulus-evoked effect was abolished, and stimulation of the septum itself did not increase NGF levels. Employing a similar intact preparation, Manseau et al. (2008) recently reported that oscillatory activity in the hippocampus could be relayed via the HS pathway to phase the firing of GABAergic neurons in the MS/DB. Hippocampal rhythmic activity of this nature would be in a position to drive NGF delivery to and release in the MS/DB.

An intriguing and striking finding in the present study was that the stimulus-evoked increase in NGF levels was stimulus pattern-dependent. In the cortex and hippocampus, GABAergic neurons are typically fast-spiking cells (Kawaguchi and Kubota, 1997; McBain and Fisahn, 2001). Among them, the majority of the fast-spiking somatostatin-containing subpopulation tends to fire in $3 \mathrm{~Hz}$ bursts of several spikes (Kawaguchi and Kubota, 1996), within the range of theta oscillations. Indeed, when simulated and applied as stimulation paradigm to the intact HS prep-

\section{$\leftarrow$}

on sIPSCS in noncholinergic MS/DB neurons $(\boldsymbol{D})$ revealed no changes in either the amplitude $(\boldsymbol{E})$ or frequency $(\boldsymbol{F})$. $\boldsymbol{G}$, Representative traces of averaged evoked IPSCs in a cholinergic MS/DB neuron during control (red), NGF (black), and wash (blue) epochs. $\boldsymbol{H}$, Histogram summarizing the mean amplitude of eIPSCs recorded in cholinergic neurons during each of the three epochs. NGF decreased the mean amplitude of eIPSCs (repeated-measures ANOVA, ${ }^{* *} p<0.01$ ); the mean amplitude during NGF application was significantly lower compared with control and wash periods (Bonferroni's multiple-comparison tests, ${ }^{*} p<0.05$ ). 
aration, it was this type of burst-spiking pattern of stimulation of the hippocampus that elevated NGF levels in the MS/DB. We note that our stimulation paradigm does not discriminate as to whether the observed increase in NGF levels is due to translocation or release, be it via either anterograde or retrograde mechanisms. We also note that our NGF ELISA measurements presumably included both intracellular and extracellular NGF. Future studies will continue to explore in the intact HS preparation whether stimulation of the hippocampus or fornix causes release of endogenous NGF and whether this results in modulation GABAergic neurotransmission in the MS/DB.

\section{NGF modulation of inhibitory synaptic activity in cholinergic MS/DB neurons}

Assuming that release from HS GABAergic axons into the extracellular milieu contributes to the stimulus-evoked increase in NGF levels in the MS/DB, we applied NGF exogenously to simulate stimulus-evoked increase in extracellular NGF. We found that NGF selectively suppressed inhibitory, vis-à-vis excitatory, synaptic transmission in the MS/DB in the rapid time scale that has been shown for BDNF (Levine et al., 1996, 1998; Blum and Konnerth, 2005; Cheng and Yeh, 2003, 2005). Our results point to the neurotrophin favoring a postsynaptic mechanism; NGF attenuated GABA-evoked current responses and decreased the amplitude of sIPSCs and eIPSCs without altering the frequency of sIPSCs. Exogenously applied GABA most likely activates both synaptic and extrasynaptic $\mathrm{GABA}_{\mathrm{A}}$ receptors, and it is possible for NGF signaling to modulate the function of both $G_{A B A}$ receptor pools. In this light, ongoing studies are investigating whether NGF modulates a recently uncovered $G_{A B A}$ receptor-mediated tonic conductance in the MS/DB. We are also assessing the existence of an ambient presence of NGF in the MS/DB, and whether it tonically dampens GABAergic neurotransmission in cholinergic MS/DB neurons. Consistent with this notion, results of pilot experiments suggest that applying the NGF scavenger protein TrkA-Fc alone $(5 \mu \mathrm{g} / \mathrm{ml})$ enhanced GABA-mediated responses in cholinergic MS/DB neurons (L. Guo and H. H. Yeh, unpublished observation).

An issue not addressed in the present study is whether intrahippocampal stimulation induced the release of mature NGF, pro-NGF, or both. Although somewhat controversial, activitydependent neuronal release of both mature and precursor forms of neurotrophins from central neurons has been reported (Lessmann et al., 2003; Bruno and Cuello, 2006; Matsumoto et al., 2008). Considering that our experiments were conducted using the $2.5 \mathrm{~S}$ form of NGF, the involvement of mature NGF is likely. Nonetheless, this does not preclude a role for pro-NGF, as both are biologically active (Lu et al., 2005).

\section{Functional implications of anterograde NGF action}

What might be the physiological relevance of anterogradely transported NGF from the hippocampus to the MS/DB? The hippocampal GABAergic projection neurons constitute the major, if not the sole, feedback loop for the inflow of activity from the MS/DB to the hippocampus. As a result, these neurons are likely under the control of powerful convergent excitatory and disinhibitory synaptic influences within the hippocampal circuit that, in turn, exert influences through feed back to the MS/DB. Such HS flow of activity has been demonstrated to phase thetalike rhythmic discharges in the MS/DB (Manseau et al., 2008) and, conceivably, could promote anterograde delivery of NGF. Since NGF is present in the GABAergic HS axons, the neurotrophin may be coreleased with GABA, although how this might occur and the underlying mechanisms remain to be demonstrated experimentally. By suppressing postsynaptic $\mathrm{GABA}_{\mathrm{A}}$ receptor function, NGF could keep in check GABA-mediated inhibition in the MS/DB. This, in combination with the previously reported direct and rapid excitatory effect on the firing activity of cholinergic neurons ( $\mathrm{Wu}$ and Yeh, 2005), serves to maintain an elevated muscarinic tone in the MS/DB and, thereby, heightened hippocampus-bound septal outflow that may underlie the long implicated role of the MS/DB in learning and mnemonic functions (Olton et al., 1978; Poucet and Herrmann, 1990; Dutar et al., 1995).

\section{References}

Acsády L, Pascual M, Rocamora N, Soriano E, Freund TF (2000) Nerve growth factor but not neurotrophin-3 is synthesized by hippocampal GABAergic neurons that project to the medial septum. Neuroscience 98:23-31.

Altar CA, DiStefano PS (1998) Neurotrophin trafficking by anterograde transport. Trends Neurosci 21:433-437.

Altar CA, Cai N, Bliven T, Juhasz M, Conner JM, Acheson AL, Lindsay RM, Wiegand SJ (1997) Anterograde transport of brain-derived neurotrophic factor and its role in the brain. Nature 389:856-860.

Blöchl A, Thoenen H (1995) Characterization of nerve growth factor (NG) release from hippocampal neurons: evidence for a constitutive and an unconventional sodium-dependent regulated pathway. Eur J Neurosci 7:1220-1228.

Blöchl A, Thoenen H (1996) Localization of cellular storage compartments and sites of constitutive and activity-dependent release of nerve growth factor (NGF) in primary cultures of hippocampal neurons. Mol Cell Neurosci 7:173-190.

Blum R, Konnerth A (2005) Neurotrophin-mediated rapid signaling in the central nervous system: mechanisms and functions. Physiology 20:70-78.

Blum R, Kafitz KW, Konnerth A (2002) Neurotrophin-evoked depolarization requires the sodium channel Nav1.9. Nature 419:687-693.

Bramham CR, Messaoudi E (2005) BDNF function in adult plasticity: the synaptic consolidation hypothesis. Prog Neurobiol 76:99-125.

Brünig I, Penschuck S, Berninger B, Benson J, Fritschy JM (2001) BDNF reduces miniature postsynaptic currents by rapid downregulation of $\mathrm{GABA}_{\mathrm{A}}$ receptor surface expression. Eur J Neurosci 13:1320-1328.

Bruno MA, Cuello AC (2006) Activity-dependent release of precursor nerve growth factor, conversion to mature nerve growth factor, and its degradation by a protease cascade. Proc Natl Acad Sci U S A 103:6735-6740.

Chen X, Ye H, Kuruvilla R, Ramanan N, Scangos KW, Zhang C, Johnson NM, England PM, Shokat KM, Ginty DD (2005) A chemical-genetic approach to studying neurotrophin signaling. Neuron 46:13-21.

Cheng Q, Yeh HH (2003) Brain-derived neurotrophic factor attenuates mouse cerebellar granule cell $\mathrm{GABA}_{\mathrm{A}}$ receptor-mediated responses via postsynaptic mechanisms. J Physiol 548:711-721.

Cheng Q, Yeh HH (2005) PLC $\gamma$ signaling underlies BDNF potentiation of Purkinje cell responses to GABA. J Neurosci Res 79:616-627.

Conner JM, Varon S (1992) Distribution of nerve growth factor-like immunoreactive neurons in the adult rat brain following colchicine treatment. J Comp Neurol 326:347-362.

Conner JM, Lauterborn JC, Yan Q, Gall CM, Varon S (1997) Distribution of brain-derived neurotrophic factor (BDNF) protein and mRNA in the normal adult rat CNS: evidence for anterograde axonal transport. J Neurosci 17:2295-2313

Davies AM (1996) The neurotrophic hypothesis: where does it stand? Philos Trans R Soc Lond B Biol Sci 351:389-394.

DiStefano PS, Friedman B, Radziejewski C, Alexander C, Boland P, Schick CM, Lindsay RM, Wiegand SJ (1992) The neurotrophins BDNF, NT-3, and NGF display distinct patterns of retrograde axonal transport in peripheral and central neurons. Neuron 8:983-993.

Du JL, Poo MM (2004) Rapid BDNF-induced retrograde synaptic modification in a developing retinotectal system. Nature 429:878-883.

Dutar P, Bassant MH, Senut MC, Lamour Y (1995) The septohippocampal pathway: structure and function of a central cholinergic system. Physiol Rev 75:393-427.

Fernandes CC, Pinto-Duarte A, Ribeiro JA, Sebastião AM (2008) Postsynaptic action of brain-derived neurotrophic factor attenuates alpha7 nico- 
tinic acetylcholine receptor-mediated responses in hippocampal interneurons. J Neurosci 28:5611-5618.

Freund TF, Buzsáki G (1996) Interneurons of the hippocampus. Hippocampus 6:347-470.

Fusco M, Oderfeld-Nowak B, Vantini G, Schiavo N, Gradkowska M, Zaremba M, Leon A (1989) Nerve growth factor affects uninjured, adult rat septohippocampal cholinergic neurons. Neuroscience 33:47-52.

Gibbs RB, Martynowski C (1997) Nerve growth factor induces Fos-like immunoreactivity within identified cholinergic neurons in the adult rat basal forebrain. Brain Res 753:141-151.

Gong S, Zheng C, Doughty ML, Losos K, Didkovsky N, Schambra UB, Nowak NJ, Joyner A, Leblanc G, Hatten ME, Heintz N (2003) A gene expression atlas of the central nervous system based on bacterial artificial chromosomes. Nature 425:917-925.

Gustilo MC, Markowska AL, Breckler SJ, Fleischman CA, Price DL, Koliatsos VE (1999) Evidence that nerve growth factor influences recent memory through structural changes in septohippocampal cholinergic neurons. J Comp Neurol 405:491-507.

Heckers S, Ohtake T, Wiley RG, Lappi DA, Geula C, Mesulam MM (1994) Complete and selective cholinergic denervation of rat neocortex and hippocampus but not amygdala by an immunotoxin against the p75 NGF receptor. J Neurosci 14:1271-1289.

Huang EJ, Reichardt LF (2001) Neurotrophins: roles in neuronal development and function. Annu Rev Neurosci 24:677-736.

Johnston MV, Rutkowski JL, Wainer BH, Long JB, Mobley WC (1987) NGF effects on developing forebrain cholinergic neurons are regionally specific. Neurochem Res 12:985-994.

Junard EO, Montero CN, Hefti F (1990) Long-term administration of mouse nerve growth factor to adult rats with partial lesions of the cholinergic septohippocampal pathway. Exp Neurol 110:25-38.

Kafitz KW, Rose CR, Thoenen H, Konnerth A (1999) Neurotrophin-evoked rapid excitation through TrkB receptors. Nature 401:918-921.

Kang HJ, Schuman EM (1995) Neurotrophin-induced modulation of synaptic transmission in the adult hippocampus. J Physiol Paris 89:11-22.

Kawaguchi Y, Kubota Y (1996) Physiological and morphological identification of somatostatin- or vasoactive intestinal polypeptide-containing cells among GABAergic cell subtypes in rat frontal cortex. J Neurosci 16:2701-2715.

Kawaguchi Y, Kubota Y (1997) GABAergic cell types and their synaptic connections in rat frontal cortex. Cereb Cortex 7:476-486.

Kawaja MD, Walsh GS, Tovich PR, Julien JP (1998) Effects of elevated levels of nerve growth factor on the septohippocampal system in transgenic mice. Eur J Neurosci 10:2207-2216.

Korsching S, Auburger G, Heumann R, Scott J, Thoenen H (1985) Levels of nerve growth factor and its mRNA in the central nervous system of the rat correlate with cholinergic innervation. EMBO J 4:1389-1393.

Korte M, Kang H, Bonhoeffer T, Schuman E (1998) A role for BDNF in the late-phase of hippocampal long-term potentiation. Neuropharmacology 37:553-559.

Lapchak PA, Araujo DM, Carswell S, Hefti F (1993) Distribution of [125I]nerve growth factor in the rat brain following a single intraventricular injection: correlation with the topographical distribution of trkA messenger RNA-expressing cells. Neuroscience 54:445-460.

Lauterborn JC, Tran TM, Isackson PJ, Gall CM (1993) Nerve growth factor mRNA is expressed by GABAergic neurons in rat hippocampus. Neuroreport 5:273-276.

Lauterborn JC, Bizon JL, Tran TM, Gall CM (1995) NGF mRNA is expressed by GABAergic but not cholinergic neurons of rat basal forebrain. J Comp Neurol 360:454-462.

Lessmann V, Gottmann K, Malcangio M (2003) Neurotrophin secretion: current facts and future prospects. Prog Neurobiol 69:341-374.

Levine ES, Dreyfus CF, Black IB, Plummer MR (1996) Selective role for trkB neurotrophin receptors in rapid modulation of hippocampal synaptic transmission. Brain Res Mol Brain Res 38:300-303.

Levine ES, Crozier RA, Black IB, Plummer MR (1998) Brain-derived neurotrophic factor modulates hippocampal synaptic transmission by increasing N-methyl-D-aspartic acid receptor activity. Proc Natl Acad Sci U S A 95:10235-10239.

Lewin GR, Barde YA (1996) Physiology of the neurotrophins. Annu Rev Neurosci 19:289-317.

Lu B, Pang PT, Woo NH (2005) The yin and yang of neurotrophin action. Nat Rev Neurosci 6:603-614.
Manseau F, Goutagny R, Danik M, Williams S (2008) The hippocamposeptal pathway generates rhythmic firing of GABAergic neurons in the medial septum and the diagonal bands: an investigation using a complex septohippocampal preparation in vitro. J Neurosci 28:4096-4107.

Matsumoto T, Rauskolb S, Polack M, Klose J, Kolbeck R, Korte M, Barde YA (2008) Biosynthesis and processing of endogenous BDNF: CNS neurons store and secrete BDNF, not pro-BDNF. Nat Neurosci 11:131-133.

McBain CJ, Fisahn A (2001) Interneurons unbound. Nat Rev Neurosci 2:11-23.

Minichiello L, Calella AM, Medina DL, Bonhoeffer T, Klein R, Korte M (2002) Mechanism of TrkB-mediated hippocampal long-term potentiation. Neuron 36:121-137.

Mizoguchi Y, Ishibashi H, Nabekura J (2003a) The action of BDNF on $\mathrm{GABA}(\mathrm{A})$ currents changes from potentiating to suppressing during maturation of rat hippocampal CA1 pyramidal neurons. J Physiol 548:703-709.

Mizoguchi Y, Kanematsu T, Hirata M, Nabekura J (2003b) A rapid increase in the total number of cell surface functional GABA(A) receptors induced by brain-derived neurotrophic factor in rat visual cortex. J Biol Chem 278:44097-44102.

Montero CN, Hefti F (1988) Rescue of lesioned septal cholinergic neurons by nerve growth factor: specificity and requirement for chronic treatment. J Neurosci 8:2986-2999.

Oliva AA Jr, Jiang M, Lam T, Smith KL, Swann JW (2000) Novel hippocampal interneuronal subtypes identified using transgenic mice that express green fluorescent protein in GABAergic interneurons. J Neurosci 20:3354-3368.

Olton DS, Walker JA, Gage FH (1978) Hippocampal connections and spatial discrimination. Brain Res 139:295-308.

Pang PT, Lu B (2004) Regulation of late-phase LTP and long-term memory in normal and aging hippocampus: role of secreted proteins tPA and BDNF. Ageing Res Rev 3:407-430.

Pascual M, Acsády L, Rocamora N, Freund TF, Soriano E (1999) Expression of neurotrophins in hippocampal interneurons immunoreactive for the neuropeptides somatostatin, neuropeptide- $\mathrm{Y}$, vasoactive intestinal polypeptide and cholecystokinin. Neuroscience 89:1089-1101.

Poucet B, Herrmann T (1990) Septum and medial frontal cortex contribution to spatial problem-solving. Behav Brain Res 37:269-280.

Pozzo-Miller LD, Gottschalk W, Zhang L, McDermott K, Du J, Gopalakrishnan R, Oho C, Sheng ZH, Lu B (1999) Impairments in high-frequency transmission, synaptic vesicle docking, and synaptic protein distribution in the hippocampus of BDNF knockout mice. J Neurosci 19:4972-4983.

Schinder AF, Poo M (2000) The neurotrophin hypothesis for synaptic plasticity. Trends Neurosci 23:639-645.

Schwab ME, Otten U, Agid Y, Thoenen H (1979) Nerve growth factor (NGF) in the rat CNS: absence of specific retrograde axonal transport and tyrosine hydroxylase induction in locus ceruleus and substantia nigra. Brain Res 168:473-483.

Segal RA (2003) Selectivity in neurotrophin signaling: theme and variations. Annu Rev Neurosci 26:299-330.

Seiler M, Schwab ME (1984) Specific retrograde transport of nerve growth factor (NGF) from neocortex to nucleus basalis in the rat. Brain Res 300:33-39.

Sobreviela T, Clary DO, Reichardt LF, Brandabur MM, Kordower JH, Mufson EJ (1994) TrkA-immunoreactive profiles in the central nervous system: colocalization with neurons containing p75 nerve growth factor receptor, choline acetyltransferase and serotonin. J Comp Neurol 350:587-611.

Takeda A, Onodera H, Sugimoto A, Kogure K, Obinata M, Shibahara S (1993) Coordinated expression of messenger RNAs for nerve growth factor, brain-derived neurotrophic factor and neurotrophin-3 in the rat hippocampus following transient forebrain ischemia. Neuroscience 55:23-31.

Tamamaki N, Yanagawa Y, Tomioka R, Miyazaki J, Obata K, Kaneko T (2003) Green fluorescent protein expression and colocalization with calretinin, parvalbumin, and somatostatin in the GAD67-GFP knock-in mouse. J Comp Neurol 467:60-79.

Tanaka T, Saito H, Matsuki N (1997) Inhibition of $\mathrm{GABA}_{\mathrm{A}}$ synaptic responses by brain-derived neurotrophic factor (BDNF) in rat hippocampus. J Neurosci 17:2959-2966.

Tóth K, Borhegyi Z, Freund TF (1993) Postsynaptic targets of GABAergic 
hippocampal neurons in the medial septum-diagonal band of Broca complex. J Neurosci 13:3712-3724.

Wardle RA, Poo MM (2003) Brain-derived neurotrophic factor modulation of GABAergic synapses by postsynaptic regulation of chloride transport. J Neurosci 23:8722-8732.

Williams LR (1991) Exogenous nerve growth factor stimulates choline acetyltransferase activity in aging Fischer 344 male rats. Neurobiol Aging 12:39-46.

Williams LR, Varon S, Peterson GM, Wictorin K, Fischer W, Bjorklund A, Gage FH (1986) Continuous infusion of nerve growth factor prevents basal forebrain neuronal death after fimbria fornix transection. Proc Natl Acad Sci U S A 83:9231-9235.

Wu CW, Yeh HH (2005) Nerve growth factor rapidly increases muscarinic tone in mouse medium septum/diagonal band of Broca. J Neurosci 25:4232-4242.
Wu YJ, Krüttgen A, Möller JC, Shine D, Chan JR, Shooter EM, Cosgaya JM (2004) Nerve growth factor, brain-derived neurotrophic factor, and neurotrophin-3 are sorted to dense-core vesicles and released via the regulated pathway in primary rat cortical neurons. J Neurosci Res 75:825-834.

Xu B, Gottschalk W, Chow A, Wilson RI, Schnell E, Zang K, Wang D, Nicol RA, Lu B, Reichardt LF (2000) The role brain-derived neurotrophic factor receptors in the mature hippocampus: modulation of long-term potentiation through a presynaptic mechanism involving TrkB. J Neurosci 20:6888-6897.

Yan Q, Rosenfeld RD, Matheson CR, Hawkins N, Lopez OT, Bennett L, Welcher AA (1997) Expression brain-derived neurotrophic factor protein in the adult rat central nervous system. Neuroscience 78:431448 